\title{
Static compensation ZMP algorithm preventing tips-over of a tele-operation excavator
}

\author{
$\underline{B o-H y u n ~ Y u}^{1 *}$, Ki-Young Park ${ }^{1}$, Kyung-Don Lee ${ }^{1}$, and Chang-Soo Han ${ }^{2}$ \\ ${ }^{1}$ Department of Robot Technology, Institute for Advanced Engineering, Young-in, Korea \\ ${ }^{2}$ Department of Mechanical Engineering, Hanyang University, An-san, Korea \\ *Corresponding author (zzigee@iae.re.kr)
}

\begin{abstract}
Purpose Research on tele-operated excavators that protect the operator from the risk of tip-over excavators in hazardous working areas has increased in popularity. The tele-operated excavator is able to protect workers from risks in hazardous working areas, but the operator cannot directly access the tip-over information from the tele-operated excavator. We propose the static compensation ZMP (Zero Moment Point) algorithm for preventing excavator tip-overs. Method Firstly, kinematic and kinetic analysis of the excavator was performed. Secondly, static compensation ZMP algorithm, which uses ZMP algorithm to determine gait stability of a biped walking robot, was developed to prevent tips-over of teleoperation excavator. Static compensation ZMP algorithm minimizes ZMP-error due to rapidly changing excavator acceleration using the center of gravity through a static compensation algorithm. Finally, the result of the proposed algorithm is simulated by RecurDyn model with Matlab Simulink co-simulation method. Results \& Discussion In the simulation result ZMP has been compared to static compensation ZMP-algorithm using an excavator dynamic model. From these results we see that the ZMP value is bigger than the static compensation ZMP. This means that the general ZMP is disturbed due to rapidly changing excavator acceleration. This problem is minimized by the static compensation ZMPalgorithm.
\end{abstract}

Keywords: excavator, tips-over, ZMP (Zero Moment Point), CoG(Center of Gravity)

\section{INTRODUCTION}

In most of construction and civil engineering works, hydraulic excavators are used. Hydraulic excavators can apply to diverse kinds of works, and compared with other equipment items, they offer high economic value and universality and thus continue to be widely used. For instance, excavators represent over 30\% of heavy equipment items that are recently being used in construction and civil engineering work sites. Excavators can apply to diverse works, and are heavily used in dangerous areas compared with other heavy equipment items. In dangerous areas, which have unconstructed environments, workers are always exposed to dangerous accidents. In actuality, most of accidents in construction works and civil engineering are involved with excavators.

To address these problems, research on teleoperation excavators is recently being conducted actively to secure the safety of workers in dangerous areas. The tele-operation excavators are operated by workers at a short distance with visual confirmation. This can secure the safety of workers, but limits the work's information on the excavator. Notably, the worker cannot feel the decline of the excavator, and thus can hardly expect the tips-over of the excavator. To address these problems, research is being actively conducted to sense the tips-over of the excavator. The research methods for sensing the tips-over of the excavator are classified into the static method designed to trace the CoG(Center of Gravity) of the excavator and expect the tips-over, and into the ZMP (Zero Moment Point) method which considers moment.

First, the static method, which uses the CoG of excavator, is to trace the excavator's CoM(Center of Mass) changing to its behaviors and to confirm whether the excavator is deviated from the support polygon. However, if the excavator experiences a sudden behavior change, kinematic causes cannot be considered and thus errors are created between the CoM and the calculated CoG.

To address these problems, research is being conducted to apply ZMP - which is used to assess the behavioral stability in biped walking robots ${ }^{1-3}$ - to excavators ${ }^{4}$. The ZMP algorithm can consider the kinetic features associated with a sudden excavator behavior in tracing CoM, thus securing more accurate traceability than the method of tracing CoG.

However, excavators, which use high-power hydraulic cylinders, generate big shocks in the driving unit when it experiences behavioral changes, compared with biped walking robots. This shock can be seen as force, which means a sudden acceleration change.

The conventional ZMP algorithm, which uses each link's CoM(Center of Mass) acceleration in the Cartesian coordinate space in tracing the entire excavator CoM, very sensitively responds to acceleration changes. Thus, the existing ZMP algorithm - compared with when it is used in biped walking robots - 
would create great accuracy error in hydraulic excavators which experience sudden acceleration changes.

To address these problems, this study proposes the static compensation ZMP algorithm by which the static method designed for tracing CoG less affected by sudden acceleration changes is compensated with ZMP algorithm so to remain robust to acceleration changes associated with shocks.

To verify the proposed algorithm, the kinetic analysis tool RecurDyn is used to create an excavator model, and the created model is linked with Mathworks' Simulink so as to develop the co-simulation environment. Thus, the algorithm that remains robust to acceleration changes due to shocks was simulated.

\section{KINEMATIC ANALYSIS}

\section{Excavator Kinematics Analysis}

Generally, excavator stability is determined by its total CoM. The excavator can have support polygon and stabilize itself if its total CoM remains within its support polygon such as floor contact area. However, the excavator will lose the floor reaction and its CoM deviates from its support ploygon. This state is defined as the excavator's unstable state. Excavator stability analysis concerns the discovery of its total CoM position.

In this paper, the excavator working system is assumed as a rigid body, and the excavator cabin's swing motion is not considered. In order to express the excavator behavior on the Cartesian coordinate ${ }^{5}$, the excavator coordinate system is set as in the Figure 1.

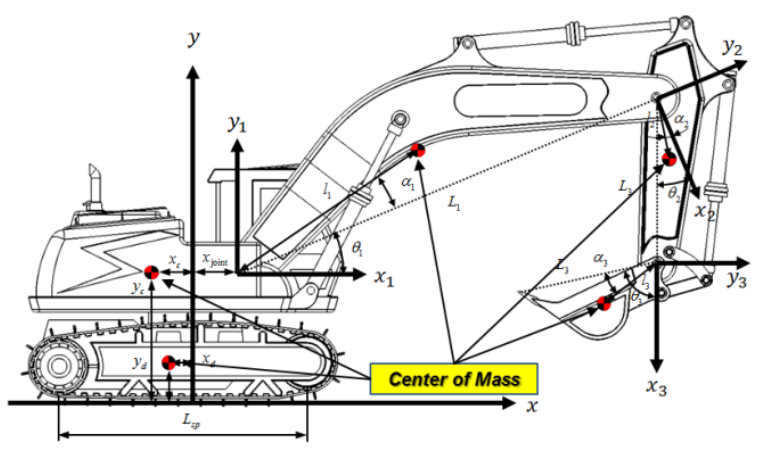

Fig.1. Excavator Model Coordinate System

This can be expressed in terms of $\mathrm{D}-\mathrm{H}$ parameters as in Table 1.

Table 1. Excavator Model D-H Parameters

\begin{tabular}{|c|c|c|c|c|}
\hline$i$ & $a_{i-1}$ & $\alpha_{i-1}$ & $d_{i}$ & $\theta_{i}$ \\
\hline 1 & 0 & 0 & 0 & $\theta_{1}$ \\
\hline 2 & $L_{1}$ & 0 & 0 & $\theta_{2}$ \\
\hline 3 & $L_{2}$ & 0 & 0 & $\theta_{3}$ \\
\hline 4 & $L_{3}$ & 0 & 0 & 0 \\
\hline
\end{tabular}

In the above D-H Parameters, the motion of the working system its location and posture, which consists of the boom, arm and bucket, is expressed in terms of each displacement function of working systems as in (1).

$$
\begin{aligned}
&{ }_{i}^{i-1} T= \operatorname{Rot}\left(\widehat{x}_{i-1}, \alpha_{i-1}\right) \operatorname{Trans}\left(\hat{x}_{i-1}, a_{i-1}\right) \\
& \operatorname{Rot}\left(\widehat{z}_{i}, \theta_{i}\right) \operatorname{Trans}\left(\bar{z}_{i}, d_{i}\right) \\
&{ }_{4}^{0} T=\left[\begin{array}{cccc}
c_{123} & -s_{123} & 0 & L_{1} c_{1}+L_{2} c_{12}+L_{3} c_{123} \\
s_{123} & c_{123} & 0 & L_{1} s_{1}+L_{2} s_{12}+L_{3} s_{123} \\
0 & 0 & 1 & 0 \\
0 & 0 & 0 & 1
\end{array}\right]
\end{aligned}
$$

The bucket end-effect's locations, namely, $p_{x}, p_{y}, \phi$ are created as in (2).

$$
\begin{aligned}
& P_{x}=l_{1} c_{1}+l_{2} c_{12}+l_{3} c_{123} \\
& P_{y}=l_{1} s_{1}+l_{2} s_{12}+l_{3} s_{123} \\
& \phi=\theta_{1}+\theta_{2}+\theta_{3}
\end{aligned}
$$

If the final excavator joint angles, namely, $\theta_{1}, \theta_{2}, \theta_{3}$ are given, each axis' arbitrary location can be evaluated, using the above expression.

\section{Cylinder Kinematics Analysis}

Using the kinematic analysis, the relational expression was determined between the excavator's joint coordinate and Cartesian coordinate. However, the hydraulic excavator behavior is driven by the hydraulics cylinders attached to the boom, arm and bucket. Thus, in order to know the joint angle, the relational expression between the joint and cylinder is needed. Figure 2 show the coordinate system and parameters defined to evaluate the relational expression between the boom and the hydraulic cylinder.

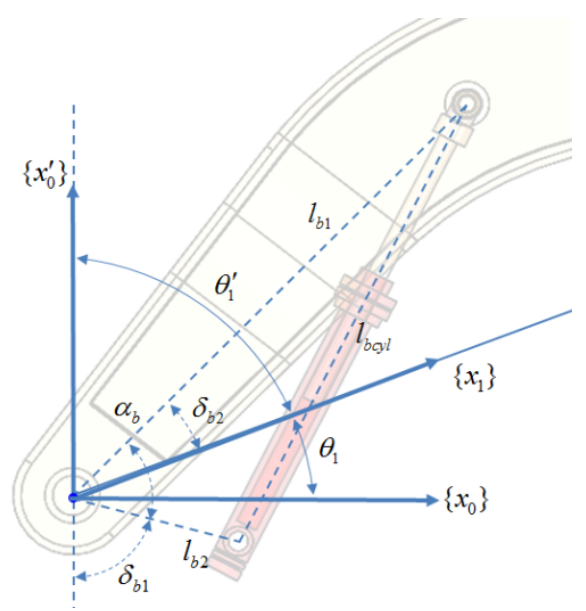

Fig. 2. Boom Cylinder Coordinate and Parameters

According to the second cosine law, the cylinder and boom kinematic parameters can be defined as in (3).

$$
l_{b c y l}^{2}=l_{b 1}^{2}+l_{b 2}^{2}-2 l_{b 1} l_{b 2} \cos \alpha_{b}
$$


Thus, the angle $\alpha_{b}$ created by the cylinder can be defined as in (4).

$$
\begin{aligned}
& \cos \alpha_{b}=\frac{l_{b 1}{ }^{2}+l_{b 2}{ }^{2}-l_{b c y l}{ }^{2}}{2 l_{b 1} l_{b 2}} \\
& \alpha_{b}=\cos ^{-1}\left(\frac{l_{b 1}{ }^{2}+l_{b 2}{ }^{2}-l_{b c y l}{ }^{2}}{2 l_{b 1} l_{b 2}}\right)
\end{aligned}
$$

Finally, using the relationship between the boom and the hydraulic cylinder, the following relational expression (5) between the hydraulic cylinder displacement and joint angle can be evaluated.

$$
\begin{aligned}
& \pi=\theta_{1}-\delta_{b 2}+\alpha_{b}+\delta_{b 1} \\
& \therefore \theta_{1}=\pi+\delta_{b 2}-\alpha_{b}-\delta_{b 1}
\end{aligned}
$$

Figure 3 shows the coordinate system and parameters defined to evaluate the relational expression between the arm and the hydraulic cylinder.

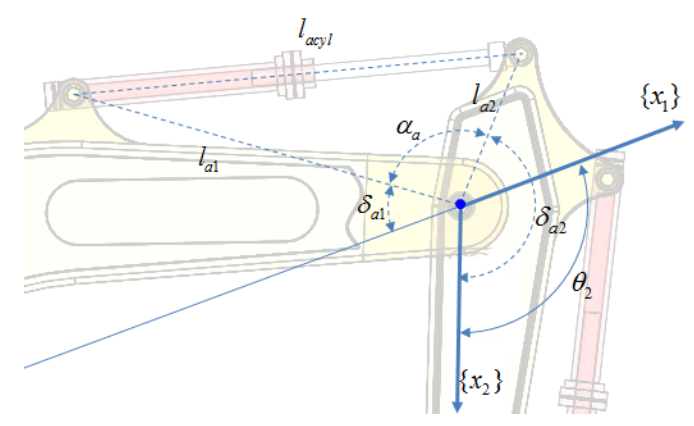

Fig.3. Arm Cylinder Coordinate and Parameters

The arm has a similar structure to the boom, so in the same method as with the boom, its rotational relationship between the hydraulic cylinder and the joint angle can be evaluated. The result is as per the following.

$$
\begin{aligned}
& l_{a c y l}{ }^{2}=l_{a 1}{ }^{2}+l_{a 2}{ }^{2}-2 l_{a 1} l_{a 2} \cos \alpha_{a} \\
& \cos \alpha_{a}=\frac{l_{a 1}{ }^{2} l_{a 2}{ }^{2}-l_{a c y l}{ }^{2}}{2 l_{a 1} l_{a 2}} \\
& \alpha_{a}=\cos ^{-1}\left(\frac{l_{a 1}{ }^{2} l_{a 2}{ }^{2}-l_{a c y l}{ }^{2}}{2 l_{a 1} l_{a 2}}\right) \\
& \pi=\delta_{a 1}+\alpha_{a}+\delta_{a 2}-\theta_{2} \\
& \therefore \theta_{2}=\delta_{a 1}+\alpha_{a}+\delta_{a 2}-\pi
\end{aligned}
$$

Figure 4 shows the coordinate system and parameters defined to evaluate the relational expression between the bucket and the hydraulic cylinder. The bucket has a four link structure, so it needs a different method from the previous one.

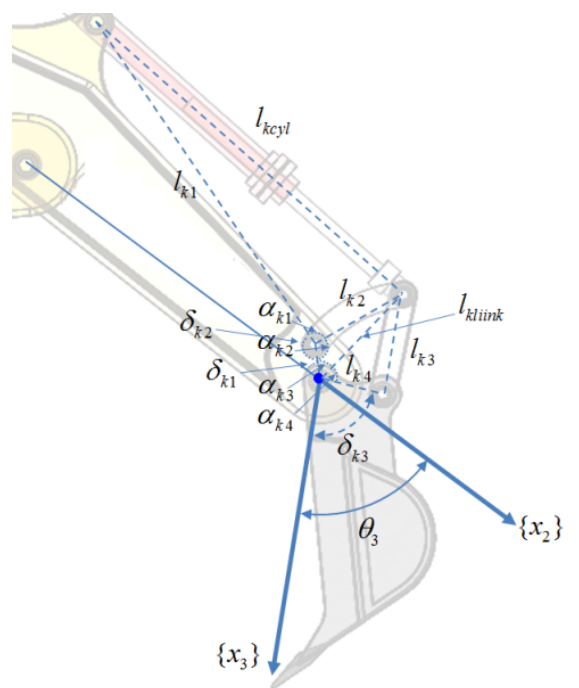

Fig.4. Bucket Cylinder Coordinate and Parameters

First, the hydraulic cylinder and the four-bar linkage start area can be evaluated with the same method as the boom. The result is shown as in (7).

$$
\begin{aligned}
& l_{k c y l}{ }^{2}=l_{k 1}{ }^{2}+l_{k 2}{ }^{2}-2 l_{k 1} l_{k 2} \cos \alpha_{k 1} \\
& \cos \alpha_{k 1}=\frac{l_{k 1}^{2} l_{k 2}^{2}-l_{k c y l}{ }^{2}}{2 l_{k 1} l_{k 2}} \\
& \alpha_{k 1}=\cos ^{-1}\left(\frac{l_{k 1}{ }^{2} l_{k 2}{ }^{2}-l_{k c y l}{ }^{2}}{2 l_{k 1} l_{k 2}}\right)
\end{aligned}
$$

Using the kinematic constraint conditions, the rotational value $\alpha_{k 2}$, which changes according to the hydraulic cylinder, can be defined as in (8).

$$
\begin{aligned}
& 2 \pi=\alpha_{k 2}+\alpha_{k 1}+\beta_{k 2} \\
& \alpha_{k 2}=2 \pi-\alpha_{k 1}-\beta_{k 2}
\end{aligned}
$$

The four-bar linkage can be defined as follows using the second cosine law, and thus all rotations within the four-bar linkage can be defined as in (9).

$$
\begin{aligned}
& l_{k \text { link }}=\sqrt{l_{k 2}{ }^{2}+l_{k 5}{ }^{2}-2 l_{k 2} l_{k 5} \cos \alpha_{k 2}} \\
& a_{k 3}=\cos ^{-1}\left(\frac{l_{k l i n k}{ }^{2}+l_{k 5}{ }^{2}-l_{k 2}{ }^{2}}{2 l_{k l i n k} l_{k 5}}\right) \\
& a_{k 4}=\cos ^{-1}\left(\frac{l_{k \text { link }}{ }^{2}+l_{k 4}{ }^{2}-l_{k 3}{ }^{2}}{2 l_{k i n k} l_{k 4}}\right)
\end{aligned}
$$

Using the kinematic constraint relationships, the bucket rotation that we want to know finally was determined as in (10). 


$$
\begin{aligned}
& l_{k c y l}{ }^{2}=l_{k 1}{ }^{2}+l_{k 2}{ }^{2}-2 l_{k 1} l_{k 2} \cos \alpha_{k 1} \\
& \cos \alpha_{k 1}=\frac{l_{k 1}{ }^{2} l_{k 2}{ }^{2}-l_{k c y l}{ }^{2}}{2 l_{k 1} l_{k 2}} \\
& \alpha_{k 1}=\cos ^{-1}\left(\frac{l_{k 1}{ }^{2} l_{k 2}{ }^{2}-l_{k c y l}{ }^{2}}{2 l_{k 1} l_{k 2}}\right) \\
& 2 \pi=\beta_{k 1}+\alpha_{k 3}+\alpha_{k 4}+\beta_{k 2}-\theta_{3}+\pi \\
& \therefore \theta_{3}=\beta_{k 1}+\alpha_{k 3}+\alpha_{k 4}+\beta_{k 2}-\pi
\end{aligned}
$$

Finally, the joint velocity and acceleration relationship between cylinder and joint angle is calculated by differential method.

$$
\dot{\theta}=\frac{d \theta}{d t}, \ddot{\theta}=\frac{d \dot{\theta}}{d t}
$$

\section{Kinetic Analysis}

In order to apply ZMP for preventing excavator tipsover, firstly, we need to know CoM(Center of Mass) point acceleration each link because force and moment is a function of acceleration. Thus, the each joints expression, determined above, should be differentiated into velocity and acceleration expression for CoM point each link.

In order to map the relationship between joint coordinate and Cartesian coordinate, Jacobian should be used.

First, using the kinematic analysis, the position function $X=F(q)$ should be evaluated, and the corresponding result is partially differentiated as much as the joint variable to obtain Jacobian $J(q)$. If the result is partially differentiated again, the acceleration Jacobian $\dot{J}(q)$ can be obtained. This can be expressed in terms of expression as in (12).

$$
\begin{aligned}
& X=F(q) \\
& \dot{X}=\frac{d F(q)}{d q} \frac{d q}{d t}=J \dot{q} \\
& \ddot{X}=\dot{J} \dot{q}+J \ddot{q}
\end{aligned}
$$

Using the above Jacobian analysis, the relationship between joint coordinate and Cartesian coordinate was determined.

\section{Static Compensation ZMP}

\section{CoG (Center of Gravity)}

Generally, the excavator CoG can be calculated if all moving links are separated, and if the position and weight of separated links are known.

If the CoM(Center of Mass) position and its total weight of each link is known, CoG can be calculated on the Cartesian coordinate using the following expression.

$$
X=\frac{\sum W_{i} \times X_{i}}{\sum W_{i}}, Y=\frac{\sum W_{i} \times Y_{i}}{\sum W_{i}}, Z=\frac{\sum W_{i} \times Z_{i}}{\sum W_{i}}
$$

Each link's weight is multiplied, and the result is divided by the total weight. Then, we know the CoG entire excavator. However, this method does not consider moment effect.

\section{ZMP (Zero Moment Point)}

The general ZMP(Zero Moment Point) algorithm was used to assess the walking stability of biped walking robots. ZMP is a point on the floor where the resultant moment of the gravity, the inertial force of the system and the external force is zero ${ }^{6,7}$. The excavator coordinate system about the inertial coordinate system is defined as in Figure 5.

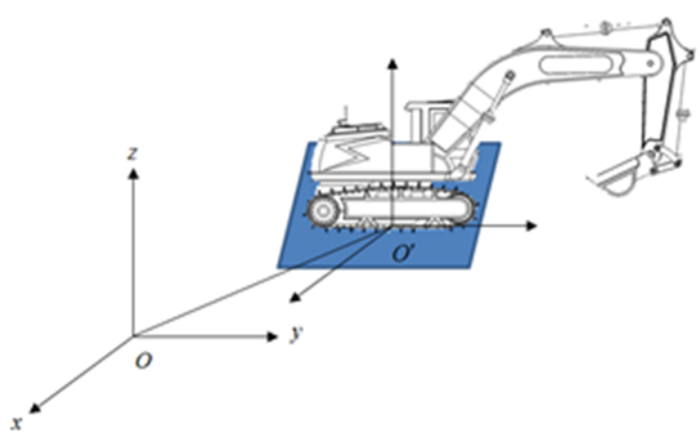

Fig.5. The Excavator Coordinate System and The Inertial Coordinate System.

The mapping between inertial coordinate and excavator coordinate is as in (14)

$$
\left(X_{Z M P}, Y_{Z M P}, 1\right)^{T}=R \times\left(X_{Z M P}^{\prime}, Y_{Z M P}, 1\right)^{T}
$$

Figure 6 shows the relationship with the arbitrary mass system in the system coordinate system.

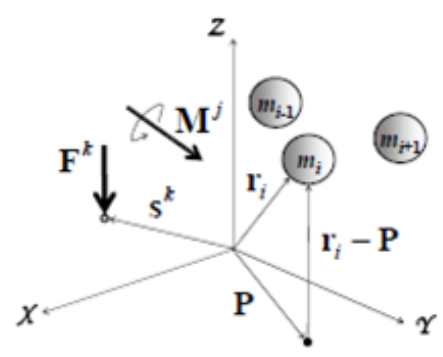

Fig.6. Definition of vectors for system

The following equation of motion at an arbitrary point on the ground $P$ is defined, which is acquired by applying d'Alembert principle. 


$$
\begin{aligned}
& \sum_{i}\left(r_{i}-P\right) \times m_{i}\left(\ddot{r}_{i}+g-\ddot{p}\right)+\rho_{c} \times m \ddot{p} \\
& +\sum_{i} T_{i}-\sum_{j} M_{j}-\sum_{k} T\left(s_{k}-p\right) \times f_{k}=M_{p}
\end{aligned}
$$

The position vector of the mass center to the point $P$ is described as in (16). If the point $P$ is ZMP, the position vector is $p_{Z M P}=\left[x_{Z M P}, y_{Z M P}, 0\right]^{T}$ and the total moment is $M_{P}=\left[0,0, M_{z}\right]^{T}$.

$$
\rho_{c}=\frac{1}{m} \sum_{m_{i}} m_{i}\left(r_{i}-p\right)
$$

Generally, Tele-operation excavator applied does not have a sensor system to measure external force because external force is very high power than biped walking robot and there is no force sensor to measure high power. If external force does not exist, can be determined as in (17), (18) in terms of $x, y$ ZMP component.

$$
\begin{aligned}
x_{Z M P}^{R}= & \frac{\sum_{i} m_{i}\left(\ddot{z}_{i}+g_{z}\right) x_{i}-\sum_{i} m_{i} \ddot{x}_{i} z_{i}}{\sum_{i} m_{i}\left(\ddot{z}_{i}+g_{z}\right) x_{i}} \\
y_{Z M P}^{R}= & \frac{\sum_{i} m_{i}\left(\ddot{z}_{i}+g_{z}\right) y_{i}-\sum_{i} m_{i} \ddot{y}_{i} z_{i}}{\sum_{i} m_{i}\left(\ddot{z}_{i}+g_{z}\right) x_{i}}
\end{aligned}
$$

The above equation, applied to the tele-operation excavator, is applied to get the position of CoG preventing excavator tips-over. Where, $m_{i}=$ each component's mass, $x_{i}, y_{i}, z_{i}=$ position coordinates value of the each component, $g_{z}=$ acceleration of gravity.

\section{Static Compensation ZMP}

Excavators, which use high-power hydraulic cylinders, generate great shocks in the driving unit according to changing behaviors. The shock created in the driving unit can be seen as force, which means a sudden acceleration change. Thus, the ZMP algorithm, which uses the acceleration, sensitively responds to acceleration changes.

In order to address these problems, we propose a method to analyze the correlation between the excavator $C o G$ and ZMP to reduce ZMP errors.

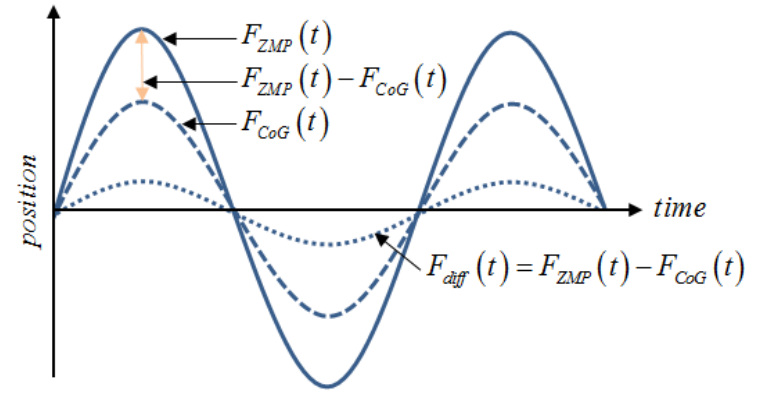

Fig.7. Concept of the Static Compensation ZMP

Figure 7 shows ZMP and CoG positions that can be obtained when the sinusoid is applied to each cylinder of the excavator according to time. If the difference between ZMP and CoG is evaluated from the above calculation, $F_{\text {diff }}(t)$ can be evaluated. Generally, due to inertial influence, ZMP value has always greater value toward the increasing movement direction than CoG value. And, the acceleration function ZMP and the position sum CoG creates great difference due to inertia, so, if the relevant system's instantaneous acceleration is determined, the maximum error scope can be defined.

By this concept, if the difference $F_{\text {diff }}(t)$ between $F_{C O G}(t)$ and $F_{Z M P}(t)$ deviates from the maximum error scope, this can be limited by cylinder maximum acceleration specification, Frequency analysis, which is low-pass-filter, apply to the $F_{\text {diff }}(t)$ function to minimize high frequency noise more than control signal. After signal processing $F_{\text {diff }}(t)$ add to $F_{C O G}(t)$. Finally, the overall ZMP error can be reduced by static compensation ZMP. Figure. 8 shows the SCZMP(Static Compensation ZMP) algorithm flow chart.

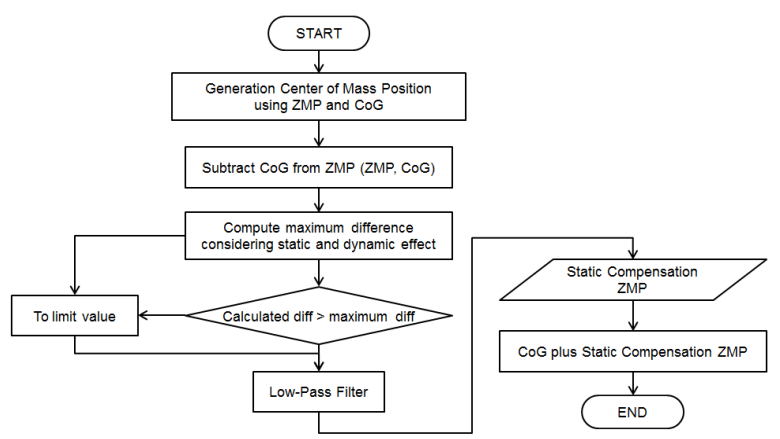

Fig. 8. Static Compensation ZMP Algorithm Flow Chart

\section{SIMULATION}

\section{Co-Simulation System}

The excavator model for simulation was created by RecutDyn Dynamic Simulator, and these parameters used for simulation model shows Table 2 . 


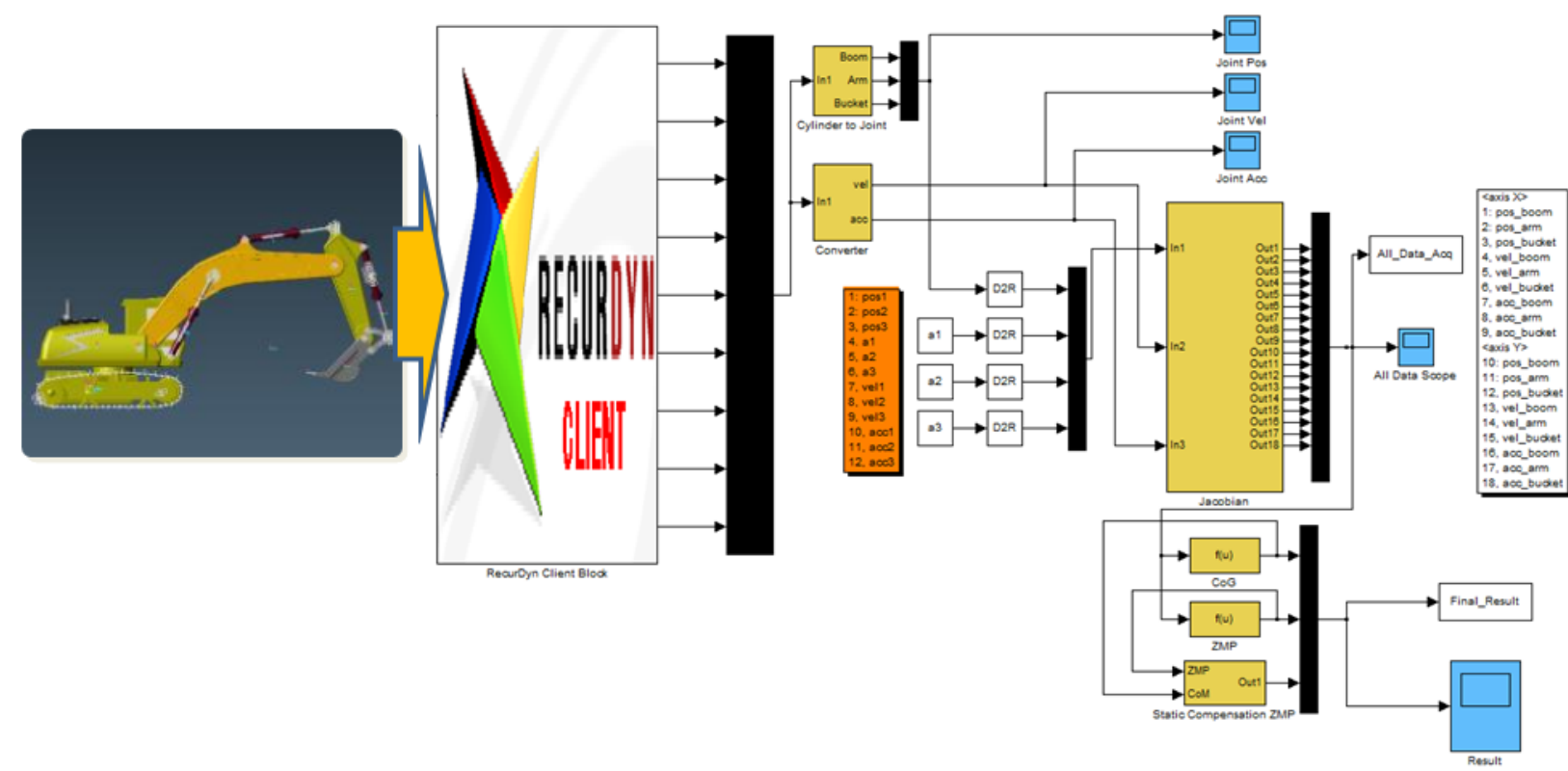

Fig.9. RecurDyn \& Matlab Simulink Co-Simulation Block Diagram for Excavator Dynamic Simulation

Table 2. Excavator Model Parameters for Simulation

\begin{tabular}{|c|c||c|c|}
\hline Parameter & Value & Parameter & Value \\
\hline \hline$L_{s p}$ & $200 \mathrm{~mm}$ & $L_{1}$ & $368.40 \mathrm{~mm}$ \\
\hline$x_{c}$ & $22.5 \mathrm{~mm}$ & $L_{2}$ & $136.59 \mathrm{~mm}$ \\
\hline$x_{y}$ & $110 \mathrm{~mm}$ & $L_{3}$ & $115.78 \mathrm{~mm}$ \\
\hline$x_{d}$ & $21 \mathrm{~mm}$ & $l_{1}$ & $160.20 \mathrm{~mm}$ \\
\hline$x_{y}$ & $35 \mathrm{~mm}$ & $l_{2}$ & $50.80 \mathrm{~mm}$ \\
\hline$m_{\text {driving }}$ & $9.11 \mathrm{~kg}$ & $l_{3}$ & $60.49 \mathrm{~mm}$ \\
\hline$m_{\text {cabin }}$ & $16.94 \mathrm{~kg}$ & $x_{\text {joint }}$ & $51.74 \mathrm{~mm}$ \\
\hline$m_{\text {boom }}$ & $7.07 \mathrm{~kg}$ & $\alpha_{1}$ & $14.70 \mathrm{deg}$ \\
\hline$m_{\text {arm }}$ & $1.69 \mathrm{~kg}$ & $\alpha_{2}$ & $10.79 \mathrm{deg}$ \\
\hline$m_{\text {bucket }}$ & $0.19 \mathrm{~kg}$ & $\alpha_{3}$ & $23.84 \mathrm{deg}$ \\
\hline & & & \\
\hline
\end{tabular}

The shock created in the driving unit, which means a sudden acceleration change, was modeled by Gaussian Distribution Noise in Matlab Simulink function block. This shock can be seen as force, which means a sudden acceleration change. The ZMP algorithm, which uses the acceleration, sensitively responds to acceleration changes.

Static compensation ZMP algorithm was developed by Matlab Simulink in Figure 9 which is very convenient method to simulate dynamic algorithm. Finally, we simulate CoG, ZMP, and Static Compensation ZMP algorithm using Co-Simulation(RecurDyn \& Matlab Simulink) method.

\section{Simulation Configuration}

Simulation input is sinusoidal signal as each cylinder velocity during 3 seconds. Table. 3 shows boom, arm, and bucket cylinder velocity input parameters.
Table 3. Excavator Cylinder Input Parameters for Stable Simulation

\begin{tabular}{|c|c|}
\hline Input Parameter & Parameters \\
\hline \hline Time & $0 \sim 3$ second \\
\hline Boom Cylinder Velocity & $-150 \sim 150 \mathrm{~mm} / \mathrm{s}$ \\
\hline Arm Cylinder Velocity & $-120 \sim 120 \mathrm{~mm} / \mathrm{s}$ \\
\hline Bucket Cylinder Velocity & $-25 \sim 25 \mathrm{~mm} / \mathrm{s}$ \\
\hline
\end{tabular}

Figure 10. Shows sequence of simulation process. Red circle is path of CoM(Center of Mass) respectively. (a) is displacement graph of boom, arm, and bucket cylinder. (b) is velocity graph of boom, arm, and bucket cylinder. (c) is acceleration graph of boom, arm, and bucket cylinder.

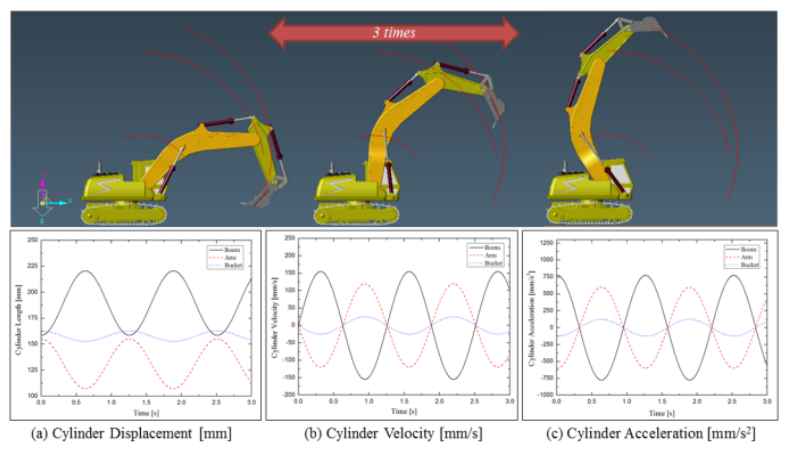

Fig.10. Excavator Simulation Sequence and Each Cylinder Displacement, Velocity, and Acceleration.

The model is carried out cylinder displacement from the input. This is converted by cylinder kinematics to each joint angle. Also, joint angular velocity and acceleration are derived from differential equation of joint angle. And then, we get the velocity and acceleration of each link CoM point on Cartesian coordinate system. Finally, we get the CoG, ZMP, and Static Compensation ZMP result. 


\section{Simulation Result}

Figure 11 is simulated by adding noise, which has Gaussian Distribution, to each joint acceleration.

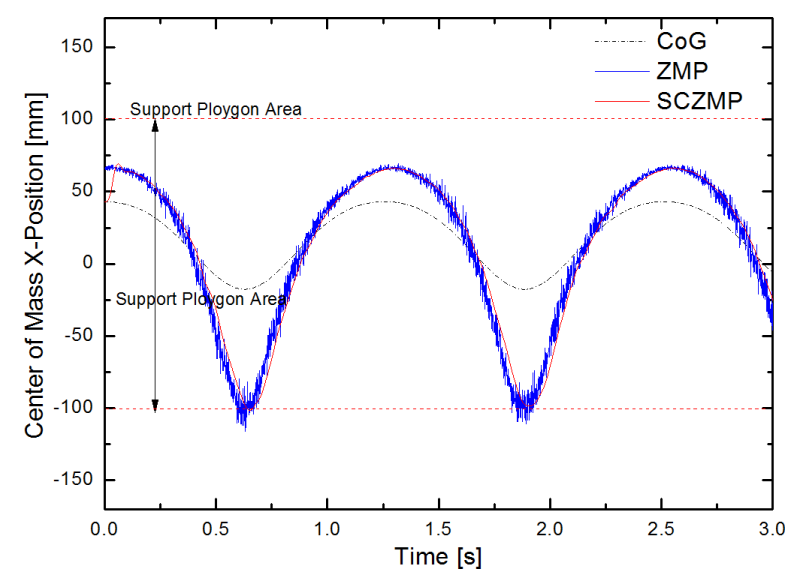

Fig.11. Stable Result with cylinder acceleration noise

From the above result, we can see that ZMP value have much noise more than SCZMP value.

To simulate excavator stability when its CoM is in the support polygon. We change simulation input parameters. Table. 4 shows simulation input parameters.

Table 4. Excavator Cylinder Input Parameters for Unstable Simulation

\begin{tabular}{|c|c|}
\hline Input Parameter & Parameter \\
\hline \hline Time & $0 \sim 3$ second \\
\hline Boom Cylinder Velocity & $-160 \sim 160 \mathrm{~mm} / \mathrm{s}$ \\
\hline Arm Cylinder Velocity & $-125 \sim 125 \mathrm{~mm} / \mathrm{s}$ \\
\hline Bucket Cylinder Velocity & $-30 \sim 30 \mathrm{~mm} / \mathrm{s}$ \\
\hline
\end{tabular}

Figure 12 shows CoM value is out of support polygon area. Figure 13 shows excavator stable, and unstable result, respectively.

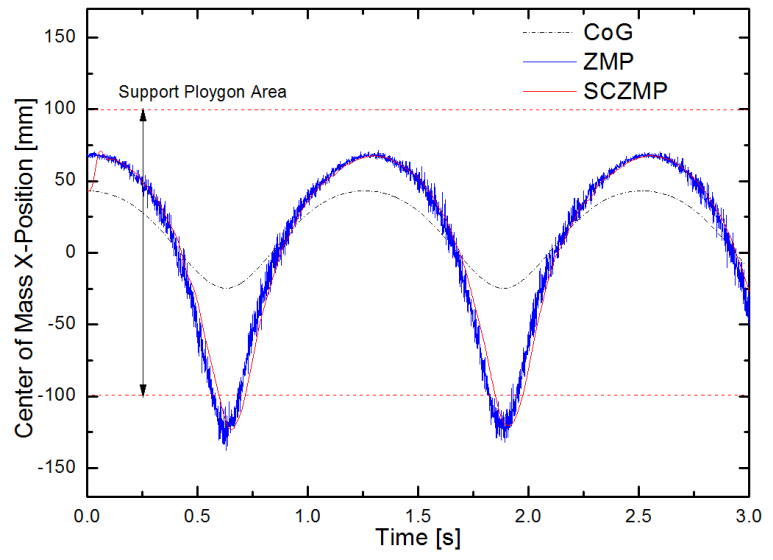

Fig.12. Unstable Result with cylinder acceleration noise

Figure 13 (a) is stable excavator motion, (b) is unstable excavator motion.

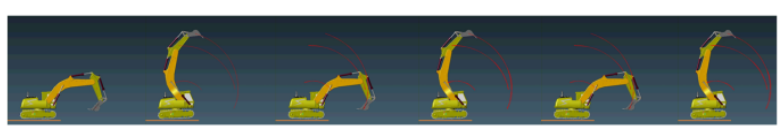

(a)

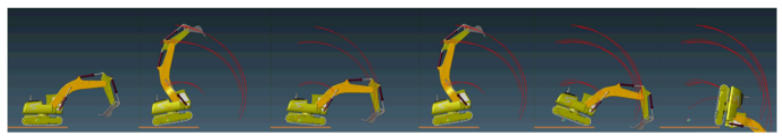

(b)

Fig.13. (a) Stable Simulation Motion, (b) Unstable Simulation Motion

\section{CONCLUSION}

In this paper we introduced Static Compensation ZMP algorithm. This algorithm can be conformed that a more accurate output, compare with general ZMP algorithm. Also we show excavator tips-over when it's total CoM out of its support polygon such as floor contact area.

\section{ACKNOWLEDGMENT}

This work was supported by the Industrial Strategic technology development program (10040180, Development of remotely piloted and optionally manned excavating system using detachable robot manipulator without any conversion of conventional excavator) funded by the Ministry of Knowledge Economy (MKE, Korea).

\section{References}

1. Kajita, S., Yokoi, K., Saigo, M., and Tanie, K., "Balancing a Humanoid Robot Using Backdrive Concerned Torque Control and Direct Angular Momentum Feedback", Proceedings of ICRA 2001, pp. 3376-3382, 2001.

2. Kajita, S., Yokoi, K., Saigo, M., and Tanie, K., "Balancing a Humanoid Robot Using Backdrive Concerned Torque Control and Direct Angular Momentum Feedback", Proceedings of ICRA 2001, pp. 3376-3382, 2001.

3. Sugihara, T., Nakamura, Y., and Inoue, H., "Realtime Humanoid Motion Generation through ZMP Manipulation based on Inverted Pendulum Control", Proceedings of ICRA 2002, pp. 1404-1409, 2002

4. Lim, T.H., Kim, Y.S., Yang, S.Y., "Development of tipping-over rate computation system for hydraulic excavator having crane function", KORUS 2004, Vol. 3, pp. 76-79, 2004.

5. Koivo, A.J., "Kinematics of excavators (backhoes) for transferring surface material", J. Aerosp.Eng., Vol. 7(1), pp. 17-31, 1994.

6. Sugano, S., Huang, Q., and Kato, I., "Stability criteria in controlling mobile robotic systems", Proceedings of the 1993 IEEE/RSJ International Conference on Intelligent Robots and Systems, Vol. 2, pp. 832838, 1993.

7. Takanishi, A., Tochizawa, M., Karaki, H., \& Kato, I. "Dynamic biped walking stabilised with optimal trunk and waist motion", Paper presented at the 
IEEE/RSJ International Workshop on Intelligent Robots and Systems, "The Autonomous Mobile Robots and Its Applications" (IROS '89), 1989. 